\title{
Biogenic synthesis of silver nanoparticles and its synergistic antimicrobial potency: an overview
}

\begin{abstract}
Recent surge in the occurrence of antimicrobial resistance genes among pathogenic microbes has been a course of concern globally. Antimicrobial resistance is one of the major threats to human health warranting research for more potent antimicrobial agents. Nanoparticles are among the most promising novel therapeutic agents that could be used against pathogens that exhibit diverse resistance to antibiotics. In particular, silver nanoparticles were shown to exhibit great antimicrobial potency. In contrast to chemical synthesis, plant extracts synthesized silver nanoparticles have no known setbacks. The extracts caused the reduction of the metallic silver, formulation and stabilization of the nanoparticles. This review has described that nanoparticles structure, particle size and its zeta $(\zeta)$ potential are among the major factor that influence its antimicrobial activity. In fact, as particle size decreases, the surface areato-volume ratio greatly increases thus effecting maximum efficacy. The review has presented in details on the biogenic synthesis of silver nanoparticles while extensively exploring the mechanisms behind such antimicrobial potency. It is hope the review will provide a reference information to both students and researchers as well as served as a guide for policy makers in decision making regarding the possibility of using silver nanoparticles as antimicrobial agents.
\end{abstract}

Keywords: antimicrobial, bacteria, biosynthesis, microorganisms, nanoparticle, silver
Volume 6 Issue I - 2019

\author{
Gumel AM,' Surayya MM, ' Yaro $M N,{ }^{2}$ Waziri \\ IZ,' Amina AA' \\ 'Department of Microbiology and Biotechnology, Federal \\ University Dutse, Nigeria \\ ${ }^{2}$ Department of Chemistry, Federal University Dutse, Nigeria
}

\begin{abstract}
Correspondence: Ahmad M Gumel, Associate Professor of Industrial Biotechnology, Department of Microbiology \& Biotechnology, Faculty of Science, Federal University Dutse, 7I56, Dutse, Jigawa State-Nigeria, Email dr.gumel@fud.edu.ng
\end{abstract}

Received: November 16, 2018 | Published: January 23, 2019

\section{Introduction}

Multidrug-resistant (MDR) bacterial pathogens have caused recent surge in hospital related comorbidities. The emergence of antibiotic resistance genesis such microorganisms has made current antibiotic therapies to become ineffective represent a growing problem, thus, becoming one of the major threats to human health. ${ }^{1}$ Antibiotic resistance is reported to be responsible for the current increase of morbidity and mortality rates. ${ }^{2}$ In bacteria, the resistance to antibiotic is a complex mechanism whose etiology highly depends on the individual species, strains and the type of resistance mechanisms developed. ${ }^{3}$ The emergence of resistance against current antibiotics mandates the need for monitoring its administration and consumption, prevention, diagnosis and rapid reduction in the abuse of the drug therapy as well as innovation and search for new and effective antimicrobial agents.

Along this line, nanotechnology and nanoparticles are among the most promising technology and novel therapeutic agents that could be used against pathogens that exhibit diverse resistance to antibiotics. ${ }^{4}$ The exotic physicochemical properties of nanoparticles have opened novel fundamental and applied frontiers that could be explored in several areas including cosmetics, environmental health, health care, food and feed, etc. ${ }^{5}$ Nanomaterials are thus seen as solution to many technological and environmental challenges in the field of solar energy conversion, catalysis, water treatment and medicine. Within the past two decades, myriads of research have documented the use of metallic nanoparticles with different morphologies, compositions and structures as antimicrobial, anti-inflmmatory agents. Additionally, they have been used bio-labeling agents and in cancer treatment. ${ }^{6}$

Though nanoparticles can be fabricated by using different physicochemical methods their synthesis by a biological method such as using microorganisms, or plant extract is a more attractive option as they are nontoxic and therefore environmentally benign especially for their application in food or as medicinal therapeutics. The approach of using plant extracts as agents of biogenic synthesis of metal nanoparticles from the salt of the corresponding metal, is considered to be the most advantageous option, simply because it is economically wise and very easy, simple, rapid and less energy extensive process since it can be carried out at room temperature. The principle behind the use of phytochemical extracts in metallic nanoparticle synthesis is said to be for bioreduction of the metal as well as stabilization of the formed nanoparticles.

The bioactive properties of several plant extracts including antimicrobial, antirheumatic, antiviral, bacterial, diuretic, expectorant, hypertensive, insecticidal etc. were reported in several literatures. Using plant extract in biogenic synthesis of nanoparticles aimed for antimicrobial potency could have a synergistic effect. This review thus highlighted on those recent cutting edge approaches and techniques applied in the biosynthesis of nanoparticles as well as their evaluation as antimicrobial agents.

\section{Nanoparticles}

Nanoparticle is a term used to describe a particle with size in the range of $1 \mathrm{~nm}-100 \mathrm{~nm}$. Due to this size range, their physic-chemical and biological properties changes fundamentally in contrast to the properties of both individual atoms and molecules and of the corresponding bulk materials. Nanoparticles can be made of materials of diverse chemical nature, ${ }^{7}$ metals, and their oxides, silicates, nonoxide ceramics, polymers, organics, carbon and biomolecules were among those explored. Nanoparticles are known to be synthesized in several different architectural morphologies such as spheres, cylinders, platelets, tubes (Figure 1) etc. 


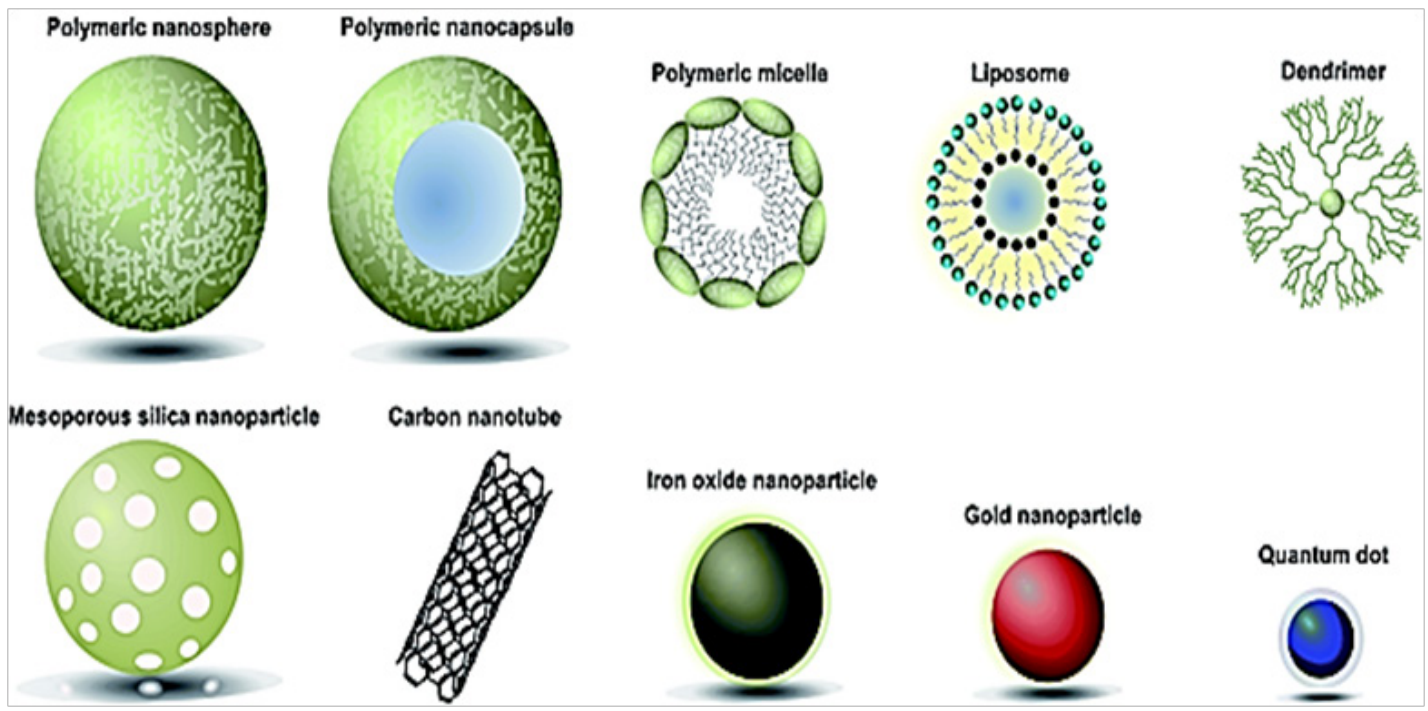

Figure I Different morphologies of nanoparticles.

Generally, depending upon the intended application, nanoparticles might be designed to have surface modifications in such a way that they are tailored to meet the needs of niche applications. This shape diversity of nanoparticles is said to be a function of chemical nature, the type of reaction medium used as well as nature or rate of dispersion, the numerous possible surface modifications the nanoparticles can be subjected to make this an important active field of research. ${ }^{8}$

There exist two main groups of nanoparticles viz, organic nanoparticles that are mostly carbon based nanoparticles (fullerenes) and the inorganic nanoparticles that include magnetic nanoparticles, noble metal nanoparticles (like gold and silver) and semi-conductor nanoparticles like those of oxides of titanium or zinc (Figure 2). There is a growing interest in inorganic nanoparticles especially those of noble metals (gold and silver) as they provide superior material properties with functional versatility thus expanding their application in medical imaging and drugs carriers for therapeutic management.

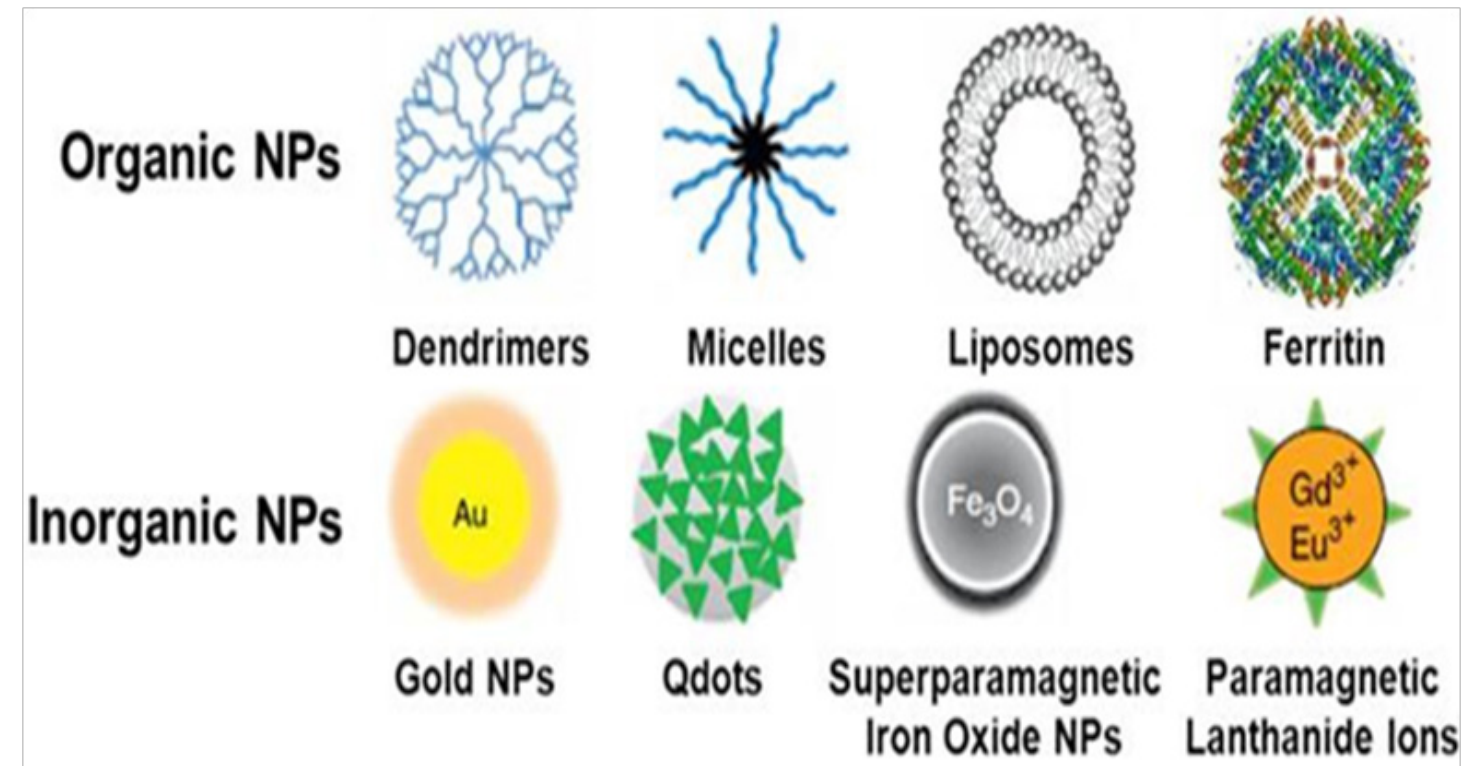

Figure 2 Typical Organic and Inorganic nanoparticles.

\section{Silver}

Silver is a basic elements that make up our planet. Being rare and naturally occurring element, silver is harder than gold with excellent ductility and malleability. Of all the known metals, pure silver is reported to has highest electrical and thermal conductivity while exhibiting lowest contact resistance. Naturally occurring silver is found in four different oxidation states: $\mathrm{Ag}, \mathrm{Ag}^{+}, \mathrm{Ag}^{2+}, \mathrm{Ag}^{3+}$, the former two are the most abundant ones, the latter are unstable in the aquatic environment. ${ }^{9}$ Metallic silver itself is insoluble in water but its salts such as those of nitrates and chloride are soluble in water.

It is a known fact that silver has antimicrobial potency thus used in surgical prosthesis and splints, fungicides and coinage. In fact, silver salts and related soluble silver based compounds were reported to be employed as therapeutic treatment in mental illness, epilepsy, nicotine addition, gastroenteritis and infectious diseases including syphilis and gonorrhea. ${ }^{9}$ The diverse application and usage of silver 
allows exposure through various routes of entry into the body, mostly by ingestion. In fact daily intake of silver was reported to stand at $70-90 \mu \mathrm{g} /$ day. Interestingly, Chen, 2008 reported that silver in any form is relatively non-toxic to immune, cardiovascular, nervous or reproductive system and it is not considered to be carcinogenic. Previous studies have demonstrated the biomedical efficacy of silver. ${ }^{10-12}$ In fact, antimicrobial properties of silver led it to be incorporated in filters to purify drinking water and clean swimming pool water.

The antimicrobial potency of silver has led its incorporation in nano-formulation. Several methods that reported the generation of nanosilver were described. Among them are spark discharging, ${ }^{13}$ electrochemical reduction, ${ }^{14}$ solution irradiation, ${ }^{15}$ cryo- chemical synthesis, ${ }^{16}$ biosynthesis ${ }^{17}$ etc. As mentioned earlier, the unique physico-chemical properties of silver nanoparticles, for instance, $\mathrm{pH}$ dependent partitioning to solid and solvation of particulate matters coupled with increase biological activities compared with the regular metal are among the reason that heightened the research interest in this area. ${ }^{18}$ All these advantages exhibited by nanoparticles were said to be owed to the higher surface area per mass, allowing a larger amount of atoms to interact with their surroundings.

\section{Biological methods for the synthesis of silver nanoparticles}

Though there established chemical processes for the synthesis of nanoparticles, unfortunately such process are debilitated by been expensive and product associated impurities. Along this line, the need for biosynthesis of nanoparticles becomes eminent. ${ }^{19}$ In contrast to chemical process, biosynthesis has none of the above limitations as an issue, simply because the nanoparticles can be synthesize biologically without the use of expensive chemicals or having toxic impurities in the synthesized products. ${ }^{20,21}$ Recent advances in biotechnology in conjunction with the robust techniques of green chemistry have made possible the use of natural reducing, capping, and stabilizing agents to prepare silver nanoparticles with desired morphology and size. The bio-redox reaction of silver ions using biomolecules found in the extracts of certain organisms is environmentally benign, yet chemically complex.

\section{Bacterial synthesis of silver nanoparticles}

Pseudomonas stutzeri AG259 is bacteria isolated from silver mine, and was reported to be the first bacteria used in synthesizing silver nanoparticles..$^{22}$ It is shown that most of these organisms can survive higher metal ion concentrations due to their resistance to that metal and the resistance is mainly due to efflux systems, and other physiological and metabolic processes like alteration of solubility and toxicity via reduction or oxidation, biosorption, bioaccumulation, extracellular complex formation or precipitation of metals, and lack of specific metal transport systems. ${ }^{23}$ The most widely accepted mechanism of silver biosynthesis is the presence of the nitrate reductase enzyme. The enzyme converts nitrate into nitrite. During in vitro synthesis of silver using bacteria, the presence of reduced form of $\alpha$-nicotinamide adenine dinucleotide phosphate (NADPH) dependent nitrate reductase would remove the downstream processing step that is required in other cases. ${ }^{24}$

\section{Myco synthesis of silver nanoparticles}

In contrast to bacteria, the ability of fungi secrete large quantity of enzymes has made it a preferred candidate for synthesizing nanoparticles at higher quantity. ${ }^{25}$ Though the exact mechanism involved in silver nanoparticle production by fungi is not fully deciphered, but the mechanism thought to follow these steps: firstly, silver ions $\mathrm{Ag}^{+}$are trapped at the surface of the fungal cells where they undergo subsequent reduction by the enzymes such as naphthoquinones and anthraquinones present in the fungal system. ${ }^{26}$ Further reduction by the NADPH-dependent nitrate reductase and a shuttle quinone extracellular process are responsible for nanoparticle formation and its stabilization. ${ }^{27}$ Despite the rapid growth of microbial cells and ease of culture, the process of microbial nanoparticles synthesis is found to be very slow process when compared to plant extracts bioreduction methods. Hence, the use of plant extracts to synthesize silver nanoparticles becomes an option that is feasible.

\section{Biogenic synthesis of silver nanoparticles using plant extracts}

The availability of plant materials, non-toxicity, and of diverse variety of metabolites that can incur silver reduction is among the major advantage of using plant extracts for the synthesis of silver nanoparticles (Figure 3). The reduction is due to phytochemicals such as terpenoids, flavones, ketones, aldehydes, amides, and carboxylic acids. Flavones, organic acids, and quinones are water-soluble phytochemicals that are responsible for the immediate reduction of the ions. Studies have revealed that xerophytes contain emodin, an anthraquinone that undergoes tautomerization leading to the formation of the silver nanoparticles. In the case of mesophytes, it was found that they contain three types of benzoquinones: cyperoquinone, dietchequinone, and remirin. It was suggested that these phytochemicals are involved directly in the reduction of the ions, formation and stabilization of the produced silver nanoparticles.

\section{Mechanism of green synthesis of silver nanoparticles}

Various plant metabolites including terpenoids, polyhydroxyphenols, carbohydrates, alkaloids, phenolic compounds, and proteins etc. are known to reduced silver ions and enhance the formation of nanoparticles. Fourier transform infrared spectroscopy (FTIR) spectroscopy of biosynthesized AgNPs has been used to demonstrate that biomolecules present in extract are responsible for synthesis of nanoparticles. Terpenoids are among the active molecule involved in biorduction of metals. It is an isoprene compound, a naturally occurring organic compound in plants, they contain fivecarbon isoprene units. In has been indicated that Geranium leaf extract contain terpenoids, which act as major player in biosynthesis of AgNPs. ${ }^{28}$ Another report confirmed that Cinnamomum zeylanicum (cinnamon) extracts contains eugenol which might be responsible for the reduction of silver nitrate to AgNPs. ${ }^{29}$ On the basis of FTIR spectroscopy data, it has been proposed that the deprotonation of the hydroxyl ion of eugenol lead to formation of resonance stabilized structures which can further be oxidized, by reducing metal ions into its nano-range. ${ }^{30}$

Besides terpenoids, flavonoids are another group of water soluble polyphenolic compounds containing 15 carbon atoms basically belonging to groups of isoflavonoids, bioflavonoids and neoflavonoids, having chelating and reducing power of metal ions. The enol and keto of flavonoids functional groups are the ones responsible for bioreduction in nanoparticle formation. ${ }^{30}$ In another study, extracts from Ocimum basilicum (sweet basil) were demonstrated to contain of flavonoids, eugenol and polyphenols that play key role in the bioreduction of silver ions to AgNPs via tautomerization of enol to keto group. ${ }^{21}$ 
In most of the related studies so far, flavonoids act as chelating agents through interaction with the metal at three positions. For example besides the bioreduction of the metal, it has been reported that quercetin, a flavonoids chelates at three positions involving the carbonyl and hydroxyls at the $\mathrm{C}_{3}$ and $\mathrm{C}_{5}$ and the catechol group at the 3' and 4' site, thus initiating nanoparticle formation (nucleation) and subsequent aggregation. In a similar study, quasi-spherical AgNPs having average diameter of 21-30 nm were synthesized using extracted apiin an apigenin glycoside of Lawsonia inermis. ${ }^{31}$ FTIR spectroscopy has revealed that a carbonyl group of apiin has been attached to the nanoparticles, demonstrating the possibility of the glycoside might participate in the reduction and stabilization of metal ions into nanoparticles. Glucose has a free aldehyde group and this can directly act as reducing agents whereas in fructose tautomerization of the keto group will caused its transition to an aldehyde thus act as antioxidants. ${ }^{21}$ Using glucose as bioreducing agent resulted in the formation of nanoparticles with different morphologies. On the other hand, using fructose in place of glucose led to the formation of monodispersed nanoparticles. ${ }^{21}$ It has been postulated that nucleophilic addition of $\mathrm{OH}^{-}$to the aldehydic group of carbohydrate oxidized it to carboxylic group, resulting in the bioreduction of the metal ions and subsequent synthesis of nanoparticles. The plant mediated synthesis is reported to take place through three main phases. Initial phase or activation phase is a phase during which metal ions are reduced and get nucleated; immediately after this phase, followed the growth phase which is characterized by spontaneous aggregation of small adjacent nanoparticles to form particles of a larger diameter, which are thermodynamically more stable. Increase in the growth phase, lead to aggregation of nanoparticles into nanotubes, nanorods and nanotriangles etc. Finally, the third phase is called the termination phase, which determines the final shape of the nanoparticles. The termination phase, the plant metabolites interact with the formed nanoparticles to undergo conformational change which is thermodynamically stable.

\section{Antibacterial activity of silver nanoparticles}

Bioefficacy of nanoparticles especially of drug resistant bacteria has proved its potential against the dilemma of effective antibiotics, thus made it to be a viable alternative. ${ }^{32}$ In particular, silver nanoparticles (AgNPs) have attracted much attention towards this end. ${ }^{33}$ Silver has always been used against various diseases; in the past it found usage as an antiseptic and antimicrobial against Gram-positive and Gramnegative bacteria ${ }^{34}$ due to its low cytotoxicity. ${ }^{35,36}$ Fayaz et al. ${ }^{17}$ have tested the synergistic antimicrobial potency of AgNPs against some Gram positive and Gram negative bacteria. The research indicated that antibacterial activities of ampicillin, kanamycin, erythromycin, and chloramphenicol were increased in the presence of AgNPs against test strains.

Despite the elaborative description of the antibacterial activity of AgNPs, their mechanism of action is still elusive. In fact, most of the so called anmicrobial efficacy seems to be owed to the multifaceted mechanism by which nanoparticles interact with the bacteria. Additionally, nanoparticle structure and its modes of establishing an interaction with bacterial cell wall may be another speculative thought on antibacterial mechanism to exploit. From a structural point of view, AgNPs are extremely of smaller dimension in the range from 1 to 100 $\mathrm{nm}$, and is a known fact that as particle size decreases, the surface area-to-volume ratio greatly increases thus assuming entirely different physico-chemical properties when compared to its bulk material of origin. On the subject of nanoparticles and bacterial interaction. several mechanisms of action have been proposed by different authors, and the most corroborated were shown in Figure $3{ }^{37}$

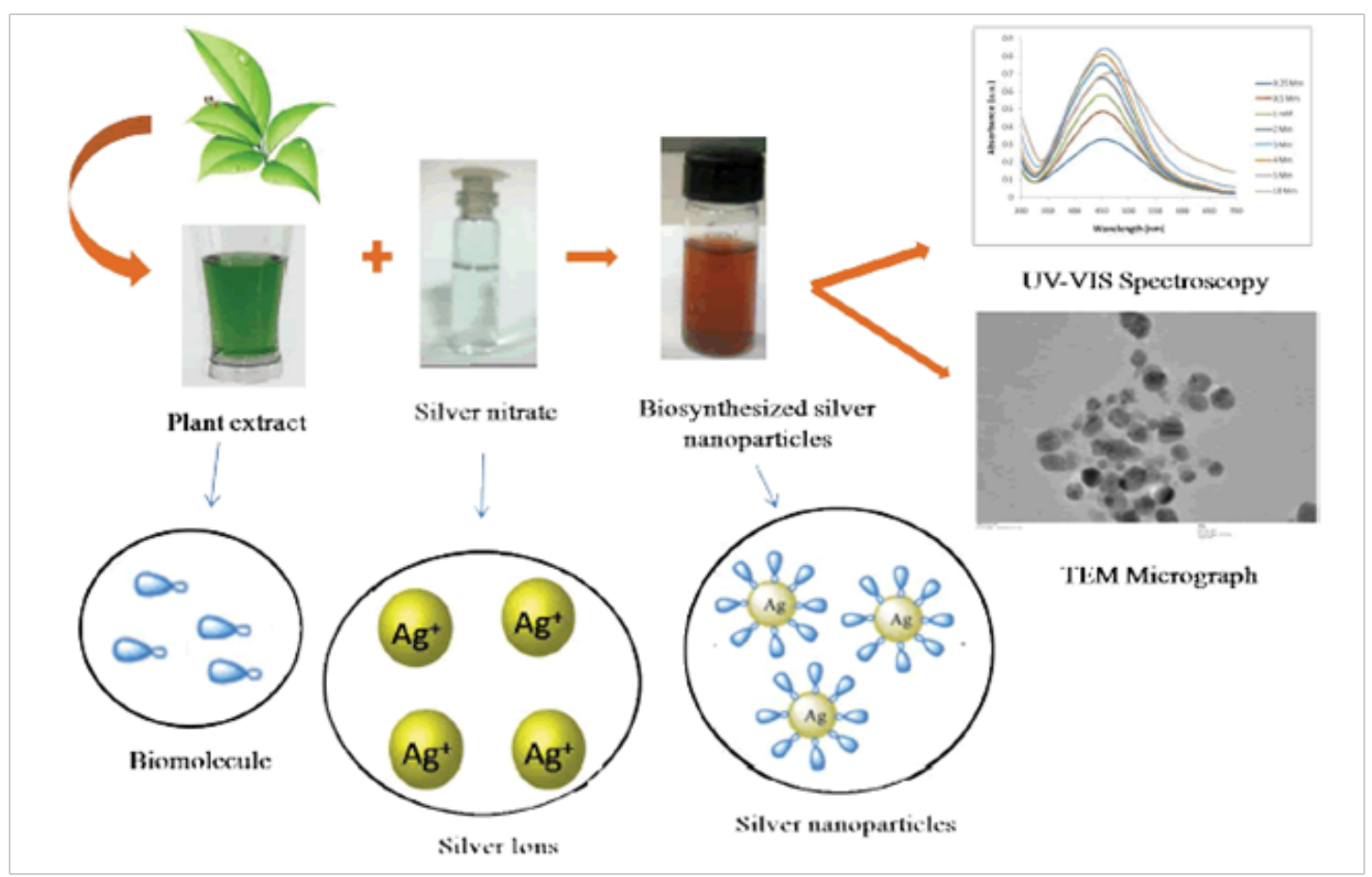

Figure 3 Steps in synthesis of silver nanoparticles using plant extracts.

Through the depicted mechanisms (Figure 4), AgNPs are able to physically interact with the cell surface of various bacteria. This is particularly important in the case of Gram-negative bacteria where numerous studies have observed the adhesion and accumulation of AgNPs to the bacterial surface. ${ }^{38,39}$ Previous study has reported that AgNPs can damage cell membranes leading to structural changes, 
which render bacteria more permeable. ${ }^{34}$ And the effect is said to be influenced by the nanoparticles' size, shape and concentration. ${ }^{40}$ In a similar study using Escherichia coli, Lazar ${ }^{34}$ confirmed that AgNPs accumulation on the membrane cell creates gaps in the integrity of the bilayer which predisposes it to a permeability increase and finally bacterial cell death. ${ }^{41}$ Several studies have shown that AgNP activity is strongly dependent on the size..$^{42}$ In fact, the bactericidal activity of AgNPs of smaller dimensions ( $<30 \mathrm{~nm}$ ) was found to be optimal against Staphylococcus aureus and Klebsiella pneumonia. ${ }^{43}$ Smaller nanoparticles seem to have a superior ability to penetrate into bacteria. In fact, the interactions with the membranes and any resulting damage, which may lead to cell death, are certainly more evident in the case of nanoparticles with smaller diameter and a positive zeta $(\zeta)$ potential.

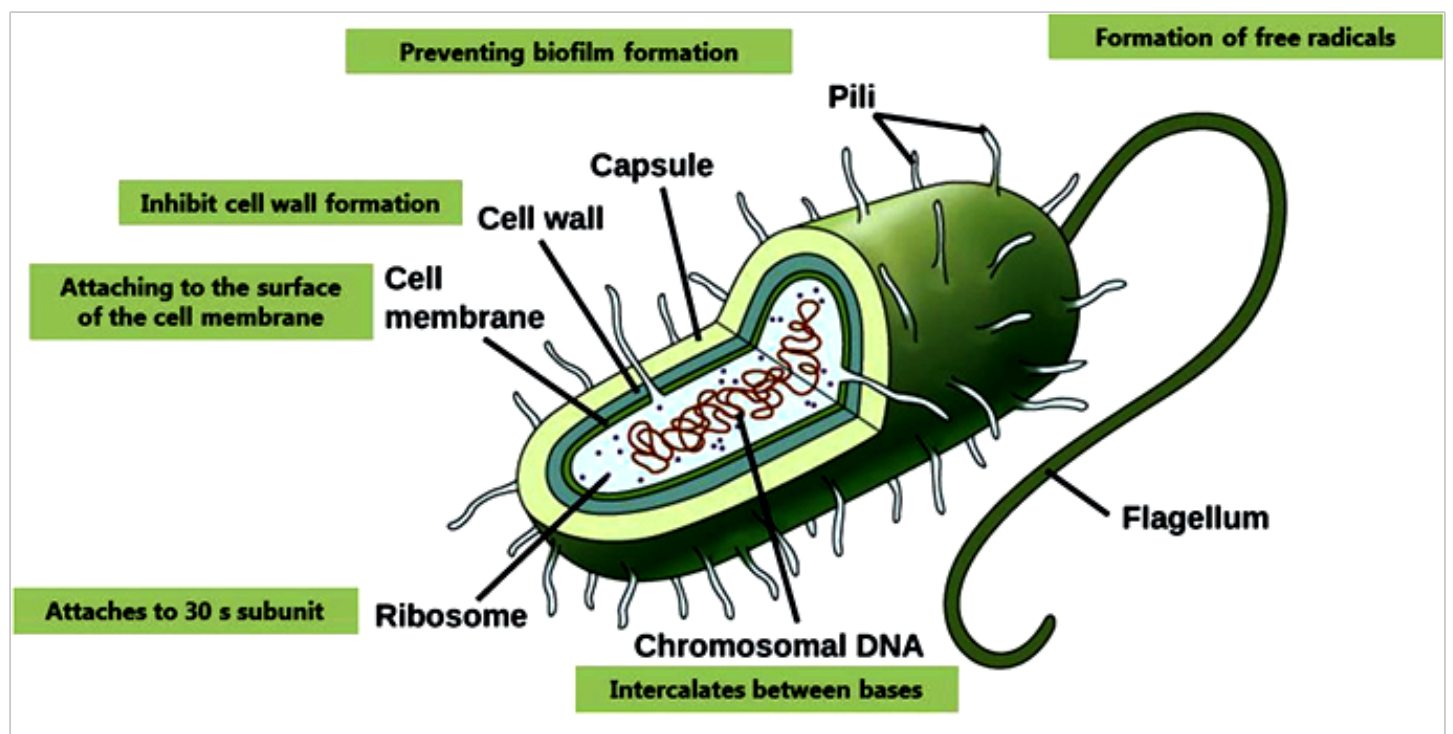

Figure 4 Proposed mechanisms of action on bacteria by silver nanoparticles.

The reason behind this phenomenal observation has been speculated to be due to electrostatic forces that develop when nanoparticles with a positive zeta potential encounter bacteria with a negative surface charge promote a closer attraction and interaction between the two entities and possibly the penetration in bacterial membranes. ${ }^{20}$ Indeed, the zeta potential along with the size of the nanoparticles are among fundamental parameters for controlling the antimicrobial activity of the nanoparticles. ${ }^{33,44}$

One should also consider that a certain amount of cationic silver is released from the nanoparticles when these are dissolved in water or when they penetrate into the cells. In effect, nanoparticles have a higher antibacterial activity than the free ions of silver, whereby the antibacterial properties are attributed to both the physical properties of nanoparticles and the elution of silver ions. ${ }^{45}$ It is thus likely that a combined effect between the activity of the nanoparticles and free ions contributes in different ways to produce a strong antibacterial activity of broad spectrum.

Furthermore, the fact that bacterial resistance to elemental silver is extremely rare ${ }^{46}$ emphasizes the presence of multiple bactericidal mechanisms that act in synergy. The silver ions bind to the protein and nucleic acid negatively charged, causing structural changes and deformations in the wall, in the membranes and in the nucleic acids of the bacterial cell. In fact, silver ions achieved its potency by interacting with a number of electron donor functional groups such as thiols, phosphates, hydroxyls, imidazoles and indoles. The AgNPs also damage membranes and induce the release of reactive oxygen species (ROS), forming free radicals with a powerful bactericidal action. ${ }^{42}$ Silver ions or small AgNPs can easily enter the microbial body causing the damage of its intracellular structures. As a consequence ribosomes may be denatured with inhibition of Protein synthesis, as well as translation and transcription can be blocked by the binding with the genetic material of the bacterial cell. ${ }^{47}$ Protein synthesis was shown to be altered by treatment with AgNPs and proteomic data have shown an accumulation of immature precursors of membrane proteins resulting in destabilization of the composition of the outer membrane. ${ }^{48}$

The correlation between the bactericidal effect and AgNP concentrations is bacterial species dependent. ${ }^{49}$ Indeed, Pseudomonas aeruginosa and Vibrio cholera were more resistant than E. coli and Salmonella typhi, but at concentrations above $75 \mu \mathrm{g} / \mathrm{mL}$, the bacterial growth was completely abolished. ${ }^{50}$ On this perspective, Hashimoto et al., ${ }^{51}$ studied AgNPs antimicrobial activity against $E$. coli and $S$. aureus showing that $E$. coli was inhibited at low concentrations, while the inhibitory effects on the growth of $S$. aureus were less marked. ${ }^{42}$ AgNPs have been shown to be definitely an effective antibiotic against E. coli, S. typhi, Staphylococcus epidermidis and $S$. aureus increasing scientific evidence has demonstrated that AgNP activity would depend not only on their concentration and size, ${ }^{52}$ but also on their shape..$^{53}$ In this regard, E. coli seems to respond better to triangular nanoparticles and is inhibited at low concentrations. Pal $\&$ Sinha $^{44}$ studied the effect of nanoparticles with spherical, rod-like and triangular shapes against $E$. coli. They showed that all of them had antimicrobial activity, with the triangular nanoparticles being qualitatively more effective. Probably the triangular shape gives a greater positive charge to the nanoparticles, which together with the active facets on a triangular-shaped particle is able to ensure a greater activity. It has been suggested that AgNPs also interfere with bacterial replication processes by adhering to their nucleic acids. This assumption, however, is controversial, because some authors denoted that AgNPs do not damage DNA,${ }^{54}$ while others ${ }^{10}$ have the notion that AgNPs intercalate into the DNA.

\section{Antifungal activity of silver nanoparticles}

In recent, extreme fungal infections have contributed in a 
significant manner to the increasing incidence of a particular disease and mortality of immune-compromised patients. ${ }^{55}$ One of the most common pathogens responsible for fungal infections is Candida species. It often causes nosocomial infection with an associated mortality rate of up to $40 \%{ }^{56} \mathrm{Kim}$ et al. ${ }^{12}$ demonstrated the antifungal activity of silver nano formulation on a total of 44 antifungal stratins of six fungal species. The literarture revealed that AgNPs are effective against C. glabrata, C. albicans, C. krusei, C. parapsilosis and T. mentagrophytes effectively. Recently studies showed that the Tulsi (Ocimum sanctum L.) mediated AgNPs exhibited antifungal activity against an opportunistic human fungal pathogen. ${ }^{57}$ Hence AgNPs is considered as potent and a fast-acting fungicide against broad spectrum of common fungi including Aspergillus, Candida and Saccharomyces.

\section{Antiviral activity of silver nanoparticles}

The cytoprotective properties of silver is well known and has been employed for the prevention of viral interaction to the host cells. ${ }^{58}$ AgNPs can also be used to prevent infection after surgery and acting as anti-HIV-1 agents. ${ }^{59}$ Therefore AgNPs interaction with microorganisms and viruses is another flourishing field of research. The studies reported that AgNPs interact with HIV-1 by binding preferentially to gp120 glycoprotein knobs. ${ }^{60}$ This sort of interaction of AgNPs specifically inhibits the binding of virus to host cells. ${ }^{61}$

\section{Conclusion}

The important qualities of any antimicrobial agents is that it should be effective, safe to use, have multiple drug targets to avoid resistance and broad actions against microbes. From the work done by previous researchers as indicated above shows that both silver nanoparticles and plant extracts possess the antimicrobial properties such as antibacterial and antifungal required for current researches to fight against infectious diseases and multidrug resistance microbes. The feasibility, safety and the advantages of using biogenic synthesis approach for nanoparticles over other methods was clearly proven to be environmentally benign and affordable.

\section{Acknowledgments}

None

\section{Conflicts of interest}

The author declares there are no conflicts of interest.

\section{References}

1. Walker B, Barrett S, Polasky S, et al. Environment Looming global-scale failures and missing institutions. Science. 2009;325:1345-1346.

2. Klevens RM, Morrison MA, Nadle J, et al. Invasive methicillinresistant Staphylococcus aureus infections in the United States. JAMA 2007;298:1763-1771.

3. Andersson DI, Hughes D. Antibiotic resistance and its cost: Is it possible to reverse resistance? Nat Rev Microbiol. 2010;8:260-271.

4. Long KS, Vester B. Resistance to linezolid caused by modification at its binding site on the ribosome. Antimicrob Agents Chemother. 2012;56:603-612.

5. Colvin VLS, Schlamp MC, Alivisatos A. Light emitting diodes made from cadmium selenide nanocrystals and a semiconducting polymer Nature. 1994;370:354-357.
6. Kumar R, Ghoshal G, Jain A, et al. Rapid Green Synthesis of Silver Nanoparticles (AgNPs) Using (Prunus persica) Plants extract: Exploring its Antimicrobial and Catalytic Activities. Journal of Nanomedicine and nanotechnology. 2017;8:4.

7. Gumel AM, Annuar MSM, Yusuf H. Perspectives on the emerging applications of multifaceted biomedical polymeric nanomaterials. Journal of Nanomaterials. 2015;16(1):310.

8. $\mathrm{Xu} \mathrm{ZP}, \mathrm{Z}$ QP, Lu GQ, et al. Inorganic Nanoparticles As Carriers For Efficient Cellular Delivery. Chemical Engineering Science. 2006;61:1027-1040.

9. Ramya M. Green Synthesis of Silver Nanoparticles. Int J Pharm Med Bio. 2012;1(1):55-61.

10. Martin GS, Mannino DM, Eaton S, et al. The epidemiology of sepsis in the United States from 1979 through 2000. N Engl JMed 2003;348:15461554.

11. WK, Koo HC, Kim KW, et al. Antibacterial activity and mechanism of action of the silver ion in Staphylococcus aureus and Escherichia coli. Appl Environ Microbiol. 2008;74:2171-2178.

12. Kim JS, Kuk E, Yu KN, et al. Antimicrobial effects of silver nanoparticles. Nanomedicine. 2007;3:95-101.

13. Lara HH, Ayala-Nunez NV, Ixtepan-Turrent L, et al. Mode of antiviral action of silver nanoparticles against HIV-1. J Nanobiotechnol. 2010; $8: 1-10$.

14. Tien DC, Tseng KH, Liao CY, et al. Colloidal silver fabrication using the spark discharge system and its antimicrobial effect on Staphylococcus aureus. Medical engineering \& physics. 2008;30(8):948-952.

15. Zhang Y, Chen F, Zhuang J, et al. Synthesis of silver nanoparticles via electrochemical reduction on compact zeolite film modified electrodes. Chemical Communications. 2002;7(23):2814-2815.

16. Chen P, Song L, Liu Y, et al. Synthesis of silver nanoparticles by $\gamma$-ray irradiation in acetic water solution containing chitosan. Radiation Physics and Chemistry. 2007;76(7):1165-1168.

17. Sergeev BM, Kasaikin VA, Litmanovich EA, et al. Cryochemical synthesis and properties of silver nanoparticle dispersions stabilised by poly (2-dimethylaminoethyl methacrylate). Mendeleev communications 1999;9(4):130-132.

18. Fayaz AM, Balaji K, Girilal M, et al. Biogenic synthesis of silver nanoparticles and their synergistic effect with antibiotics: a study against gram-positive and gram-negative bacteria. Nanomedicine. 2010;6(1):103-109.

19. Lok CN, Ho CM, Chen R, et al. Proteomic analysis of the mode of antibacterial action of silver nanoparticles. $J$ Proteome Res. 2006;5(4):916-924.

20. Parasharu KS. Bioinspired Synthesis of Silver Nanoparticles. Digest Journal of Nanomaterials and Biostructures. 2009;4:159-166.

21. Begum NA, Mondal S, Basu S, et al. Biogenic synthesis of Au and Ag nanoparticles using aqueous solutions of Black Tea leaf extracts. Colloids and Surface B Biointerfaces. 2009;71(1):113-118.

22. Ahmad N, Sharma S, Alam MK, et al. Rapid synthesis of silver nanoparticles using dried medicinal plant of basil. Colloids Surf B Biointerfaces. 2010;81:81-86.

23. Haefeli C, Franklin C, Hardy K. Plasmid-determined silver resistance in Pseudomonas stutzeri isolated from a silver mine. J Bacteriol. 1984; 158:389-392

24. Husseiny MI, El-Aziz MA, Badr Y, et al. Biosynthesis of gold nanoparticles using Pseudomonas aeruginosa. Spectrochimia Acta Part A. 2007;67:1003-1006. 
25. Vaidyanathan R, Gopalram S, Kalishwaralal K, et al. Enhanced silver nanoparticle synthesis by optimization of nitrate reductase activity. Colloids Surf B Biointerfaces. 2010;75:335-341.

26. Mohanpuria P, Rana KN, Yadav SK. Biosynthesis of nanoparticles: technological concepts and future applications. J Nanopart Res. 2008;10: 507-517.

27. Mukherjee P, Ahmad A, Mandal D, et al. Fungus mediated synthesis of silver nanoparticles and their immobilization in the mycelial matrix: a novel biological approach to nanoparticle synthesis. Nano Lett. 2001;1:515-519.

28. Jha AK, Prasad K, Prasad K, et al. Plant system: nature's nanofactory. Colloids Surf B Biointerfaces. 2009;73:219-223.

29. Shankar SS, Ahmad A, Sastry M. Geranium leaf assisted biosynthesis of silver nanoparticles. Biotechnol Prog. 2003;19:1627-1631.

30. Makarov VV, Love AJ, Sinitsyna OV, et al. "Green" nanotechnologies: Synthesis of metal nanoparticles using plants. Acta naturae. 2014;6(1):35-44.

31. Sathishkumar M, Sneha K, Won SW, et al. Cinnamon zeylanicum bark extract and powder mediated green synthesis of nano crystalline silver particles and its bactericidal activity. Colloids Surf B Biointerfaces. 2009; 73:332-338

32. Kasthuri J Veerapandian S Rajendiran N. Biological synthesis of silver and gold nanoparticles using apiin as reducing agent. Colloids Surf B Biointerfaces. 2009;68:55-60.

33. Rai MK, Deshmukh SD, Ingle AP, et al. Silver nanoparticles: The powerful nanoweapon against multidrug-resistant bacteria. J Appl Microbiol. 2012;112:841-852.

34. Jana S, Pal T. Synthesis, characterization and catalytic application of silver nanoshell coated functionalized polystyrene beads. J Nanosci Nanotechnol. 2007;7:2151-2156.

35. Lazar V. Quorum sensing in Biofilms-How to destroy the bacterial citadels or their cohesion/power? Anaerobe. 2011;17:280-285.

36. Biel MA, Sievert C, Usacheva M, et al. Antimicrobial photodynamic therapy treatment of chronic recurrent sinusitis biofilms. Int. Forum Allergy Rhinol. 2011;1:329-334.

37. Dos Santos CA, Seckler MM, Ingle AP, et al. Silver nanoparticles: Therapeutical uses, toxicity, and safety issues. J Pharm Sci. 2014;103:1931-1944.

38. Chen X. Nanosilver: A Nanoproduct in Medical Applications. Toxicol Lett. 2008;76:1-12.

39. Morones JR, Elechiguerra JL, Camacho A, et al. The bactericidal effect of silver nanoparticles. Nanotechnology. 2005 Oct;16(10):2346-2353.

40. Rai M, Yada A,Gade A. Silver nanoparticles as a new generation of antimicrobials. Biotechnology advances. 2009;27(1):76-83.

41. Rai M, Kon K, Ingle A, et al. Broad-spectrum bioactivities of silver nanoparticles: The emerging trends and future prospects. Appl Microbiol Biotechnol. 2014;98:1951-1961.

42. Saadabi AMA, Ali NM, Mohammed HI, et al. An in vitro antimicrobial activity of Calotropis procera (Ait.) R.Br. extracts on certain groups of pathogenic microorganisms. Res J Med Sci. 2012;6(1):13-17.

43. Chernousova S, Epple M. Silver as antibacterial agent: Ion, nanoparticle, and metal. Angew Chem Int Ed Engl. 2013;52:1636-1653.
44. Sweet MJ, Chesser A, Singleton I. Review: Metal-based nanoparticles; size, function, and areas for advancement in applied microbiology. Adv Appl Microbiol. 2012;80:113-142.

45. Pal G, Sinha NK. Isolation, crystallization, and properties of calotropins DI and DII from Calotropis gigantean. Arch Biochem Biophys. 1980;202(2):321-329.

46. Meire MA, Coenye T, Nelis HJ, et al. Evaluation of Nd:YAG and Er: YAG irradiation, antibacterial photodynamic therapy and sodium hypochlorite treatment on Enterococcus faecalis biofilms. Int Endod J. 2012;45:482-491.

47. Wu D, Fan W, Kishen A, et al. Evaluation of the antibacterial efficacy of silver nanoparticles against Enterococcus faecalis biofilm. J Endod. 2014;40(2):285-290.

48. Collins TL, Markus EA, Hassett DJ, et al. The effect of a cationic porphyrin on Pseudomonas aeruginosa biofilms. Curr Microbiol. 2010; 61:411-416.

49. Zhang M, Zhang K, De Gusseme B, et al. The antibacterial and antibiofouling performance of biogenic silver nanoparticles by Lactobacillus fermentum. Biofouling. 2014;30:347-357.

50. Jain J, Arora S, Rajwade JM, et al. Silver nanoparticles in therapeutics: Development of an antimicrobial gel formulation for topical use. Mol Pharm. 2009;6:1388-1401.

51. Periasamy S, Joo HS, Duong AC, et al. How Staphylococcus aureus biofilms develop their characteristic structure. Proc Natl Acad Sci USA. 2012;109:1281-1286.

52. Hashimoto MC, Prates RA, Kato IT, et al. Antimicrobial photodynamic therapy on drug-resistant Pseudomonas aeruginosa-induced infection. An in vivo study. Photochem Photobiol. 2012;88:590-595.

53. Lu Z, Dai T, Huang L, et al. Photodynamic therapy with a cationic functionalized fullerene rescues mice from fatal wound infections. Nanomedicine. 2010;5:1525-1533.

54. Choi O, Hu Z. Size dependent and reactive oxygen species related nanosilver toxicity to nitrifying bacteria. Environ Sci Technol. 2008;42:4583-4588.

55. Silver S. Bacterial silver resistance: Molecular biology and uses and misuses of silver compounds. FEMS Microbiol Rev. 2003;27:341-353.

56. Panacek A, Kolar M, Vecerova R, et al. Antifungal activity of silver nanoparticles against Candida spp. Biomaterials. 2009;30:6333-6340.

57. Khatoon N, Mishra A, Alam H, et al. Biosynthesis, characterization, and antifungal activity of the silver nanoparticles against pathogenic candida species. BioNanoSc. 2010;5:65-74.

58. Sun RWY, Chen R, Chung NPY, et al. Silver nanoparticles fabricated in Hepes buffer exhibit cytoprotective activities toward HIV-1 infected cells. Chem Comm. 2005;28(40):5059-5061.

59. Elechiguerra JL, Burt JL, Morones JR, et al. Interaction of silver nanoparticles with HIV-1. J Nanobiotechnol. 2005;3:1-10.

60. Song JY, Kim BS. Rapid biological synthesis of silver nanoparticles using plant leaf extracts. Bioprocess Biosyst Eng. 2009;32(1): 79-84.

61. Becker RO. Silver ions in the treatment of local infections. Metal-based drugs. 1999;6:311. 